\title{
Correction to: Liposomal Carrier Conjugated to APP-Derived Peptide for Brain Cancer Treatment
}

\author{
Martin Gabay ${ }^{1} \cdot$ Abraham Weizman $^{2,3}$ (1) $\cdot$ Nidal Zeineh $^{1} \cdot$ Meygal Kahana $^{1} \cdot$ Fadi Obeid $^{1} \cdot$ Nahum Allon $^{1}$. \\ Moshe Gavish ${ }^{1}$ (D)
}

Published online: 21 December 2021

(c) Springer Science+Business Media, LLC, part of Springer Nature 2021

\section{Correction to:}

\section{Cellular and Molecular Neurobiology} (2021) 41:1019-1029

https://doi.org/10.1007/s10571-020-00969-1

The original version of this article unfortunately contained an error in the text under Materials and Methods section.

The sentence "The conjugated targeting peptide (1,2-dioleoyl-sn-glycero-3-succinate-Cys-HisLeu-Asp-Ile-Ile-Trp$\mathrm{COOH})$ " should read as "The conjugated targeting peptide (1,2-dioleoyl-sn-glycero-3-succinate-Ala-His-Arg-Glu-ArgMet-Ser-COOH)".

The original article has been corrected.

The original article can be found online at https://doi.org/10.1007/ s10571-020-00969-1.

Moshe Gavish

mgavish@technion.ac.il

1 The Ruth and Bruce Rappaport Faculty of Medicine, Technion Institute of Technology, 31096 Haifa, Israel

2 Research Unit, Geha Mental Health Center and the Laboratory of Biological Psychiatry, Felsenstein Medical Research Center, 4910002 Petah Tikva, Israel

3 Sackler Faculty of Medicine, Tel Aviv University, 6997801 Tel Aviv, Israel
Publisher's Note Springer Nature remains neutral with regard to jurisdictional claims in published maps and institutional affiliations. 